\title{
Improving Poor GPS Area Localization for Intelligent Vehicles
}

\author{
Dinh-Van Nguyen, Fawzi Nashashibi \\ RITS Team, INRIA \\ Paris, France \\ Dinh-van.nguyen@inria.fr
}

\author{
Trung-Kien Dao, Eric Castelli \\ MICA Institute (HUST - CNRS/UMI2954 - Grenoble \\ INP), Hanoi University of Science and Technology \\ Hanoi, Vietnam \\ Trung-kien.dao@mica.edu.vn
}

\begin{abstract}
Precise positioning plays a key role in successful navigation of autonomous vehicles. A fusion architecture of Global Positioning System (GPS) and Laser-SLAM (Simultaneous Localization and Mapping) is widely adopted. While Laser-SLAM is known for its highly accurate localization, GPS is still required to overcome accumulated error and give SLAM a required reference coordinate. However, there are multiple cases where GPS signal quality is too low or not available such as in multi-story parking, tunnel or urban area due to multipath propagation issue etc. This paper proposes an alternative approach for these areas with WiFi Fingerprinting technique to replace GPS. Result obtained from WiFi Fingerprinting will then be fused with LaserSLAM to maintain the general architecture, allow seamless adaptation of vehicle to the environment.
\end{abstract}

Keywords-GPS; Laser; SLAM; WiFi Fingerprinting; Fusion; Localization; Intelligent Vehicle; Autonomous Vehicle; Particle Filter.

\section{INTRODUCTION}

A fusion system of Laser-SLAM and GPS is commonly adopted for localization of smart vehicle. Since Laser-SLAM is only capable of delivering accurate localization result within its local coordinate system, GPS information is required to map SLAM coordinate to a global one. Also, Laser-SLAM motion model is prone to accumulated error after a long run. Thus a combination with global GPS position will not only help to improve positioning results but also potentially solve SLAM problem of loop closure [1],[2], [3]. Still, there are areas with low to no GPS signal such as Multi-story parking, tunnel or urban area with dense construction. Hence, it is necessary to find an alternative approach for these areas.

A lot of studies recently address this issue with different approaches. Studies in [2], [4], [5] make use of environment static map to improve SLAM matching confidence. A network of cooperating vehicles is explored in [6], [7] with the aim to improve localization of each vehicle. A complete solution utilizes a wide range of information such as GPS, radio frequency identification, vehicle to vehicle and vehicle to infrastructure communication is introduced in [8]. However, these solutions often fall into the problem of fusing different sensors data and information. Dealing with various form of information standards, errors and uncertainties bring unstable to the system as a whole. Moreover, the combination of GPS and SLAM performs well in most of the cases, a complicated addition to the system for cases like tunnel, car park should be avoided.
This paper proposes to use WiFi Fingerprinting techniques as a replacement for GPS information in weak GPS area. WiFi Fingerprinting localization is a technique where WiFi map of targeted environment will be learned in training phase. A prediction phase will carry out location by comparing current signal with pre-learned radio map. While learning radio map of the targeted area is mandatory for WiFi Fingerprinting, the effort of collecting such data is much less in comparison to map-based and camera methods. Moreover, WiFi Fingerprinting is capable of mimicking GPS behavior in the fusion solution with LaserSLAM. The fusion system of GPS and SLAM can be smoothly switched to WiFi and SLAM when certain conditions are met. This reduces uncertainty need to be added to the vehicle.

The main idea of this study is to replace poor GPS signal in certain areas with results from WiFi Fingerprinting localization method. A fusion strategy using a bootstrap particles filter of GPS - SLAM and WiFi - SLAM is also proposed. This fusion approach will help vehicles to adapt seamlessly to the change of environment.

This paper structure is as follows. In section II, WiFi Fingerprinting method using ensemble neural network is explained together with a fusion strategy. Section III describes experiments conducted. Finally, Section IV concludes the paper with expected future improvement.

\section{Methodology}

\section{A. WiFi Fingerprinting method}

WiFi fingerprinting localization is a technique based on learning the map of WiFi RSSI (Radio Signal Strength Indicator) available in the environment at multiple reference points spread across the environment. The main assumption is that each reference point has a unique pattern of RSSIs of all available Access Points (APs). This pattern then allows vehicles to recognize the location just by scanning RSSIs for next visit. This method has two main steps. The first step is a training phase with multiples WiFi scanning at each reference position in an environment is recorded together with its coordinate. The second step is a prediction phase where scanning data of RSSIs without coordinate is compared to data registered in step 1. A prediction from the second step is likely the current position of the vehicle.

The major challenge in this approach is RSSIs of standard WiFi system are often noisy due to interference, and multipath propagation problem. A raw data processing will be performed 
on noisy and unstable wifi signal strength. Upon recording a vector of RSSI and the corresponding location as in (1) where $x_{i, j}$ is WiFi RSSI from jth WiFi APs recorded in ith scan, $\rho_{l}$ is a label which has corresponding coordinate at position of sampling and $n$ is fixed constant. Here, $n$ should be greater than total number of APs in learning environment

$$
\left\{x_{i, 1}, x_{i, 2}, x_{i, 3}, \ldots, x_{i, n}, \rho_{l}\right\}
$$

Collected data will be normalized in the range of $[-1,1)$ where in particular scan, detected AP RSSI would be normalized (2) in the range $[0,1)$ with 0 as weakest possible signal strength and 1 as strongest possible signal strength. Other undetected APs at $\rho_{l}$ will take value -1

$$
x_{i}=\left\{\begin{aligned}
-1, A P_{i} \text { undetected } \\
1-\frac{(-1) \times R S S I}{100}, A P_{i} \text { detected }
\end{aligned}\right.
$$

A system of 2 omnidirectional wifi antenna is mounted close to each other to minimize the impact of signal interference or multipath problem of the radio signal. In a particular scan, the antenna with highest RSSI between the two will be recorded. This is due to the observation that interference and multipath propagation will most likely reduce received signal strength. Thus, the higher RSSI will likely to be closer to direct signal without interference.

For the second step, A set of neural networks is implemented to learn from training data and perform. However, as RSSI appears to be noisy and scanning frequency of WiFi is low in comparison to movement speed, data will be considered to be a high variant. Using a method called Ensemble Bagging (Bootstrap Aggregating) Neural Network, which is well-known for combining multiple learning models to derive better results of prediction [9], [10], the system is expected to overcome high variant and noisy data issue.

Consider a classification method with a pair $\left\{X_{i}, Y_{j}\right\}$ where $X_{i}$ is a vector of predictor variable and $Y_{j}$ denotes a response, $Y_{j} \in\{1,2, \ldots m\}$. The target function is $P(Y=j \mid X=x)$ for classification. A function estimator which results from a set of training samples and a classification model is formed (3).

$$
\left.g(\cdot)=h\left(\left(X_{1}, Y_{1}\right),\left(X_{2}, Y_{2}\right), \ldots,\left(X_{n}, Y_{m}\right)\right)\right)
$$

Bagging algorithm consists following steps:

Step 1: Construct a bootstrap sample (4) by randomly sampling with replacement $n$ times from original data:

$$
\left(X_{1}^{\wedge}, Y_{1}^{\wedge}\right),\left(X_{2}^{\wedge}, Y_{2}^{\wedge}\right), \ldots,\left(X_{n}^{\wedge}, Y_{m}^{\wedge}\right)
$$

Step 2: Compute bootstrapped estimator $g^{\wedge}(\cdot)$ in (5) by applying same classification model to newly formed bootstrap sample.

$$
\left.g^{\wedge}(\cdot)=h\left(\left(X_{1}^{\wedge}, Y_{1}^{\wedge}\right),\left(X_{2}^{\wedge}, Y_{2}^{\wedge}\right), \ldots,\left(X_{\mathrm{n}}^{\wedge}, Y_{\mathrm{m}}^{\wedge}\right)\right)\right)
$$

Step 3: Repeat two steps above for $\mathrm{K}$ times with $\mathrm{K}$ is large. The bagging estimator is (6).

$$
g_{\text {bagg }}^{\wedge}(\cdot)=\frac{1}{K}\left(\sum_{i=1}^{K} g^{\wedge}(\cdot)\right)
$$

Theoretically, the bagging estimator is (7) as $\mathrm{K}$ goes to infinity:

$$
g_{\text {bagg }}^{\wedge}(\cdot)=\mathbf{E}^{\wedge}\left[g^{\wedge}(\cdot)\right]
$$

In practice, a finite large $\mathrm{K}$ is expected to improve the accuracy of Monte Carlo approximation. In this study, a model of the neural network is constructed without carefully tuning parameters. Then $\mathrm{K}$ is chosen at 100 simple neural networks for ensemble purpose.

\section{B. Fusion of WiFi Fingerprinting with Laser-SLAM}

As mentioned in section I, this study aims to maintain the architecture of fusion between GPS - SLAM for a seamless adaptation of environmental conditions. A fusion strategy using particle filter is applied to accomplish the goal. The general design is demonstrated in Fig. 1.

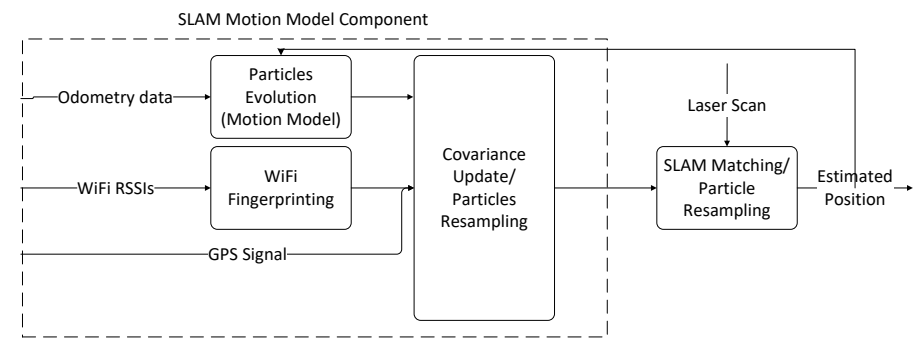

Fig. 1. Fusion architecture of WiFi/ GPS and Laser SLAM

In this solution, GPS signal / WiFi localization result with expected error will serve as variance $\sigma$ in covariance of particle estimation and $\mathrm{WiFi} /$ GPS location. Thus, taking WiFi/ GPS location as median ( $\mu_{W i F i}$ and $\mu_{G P S}$ respectively) it is possible to update the score of each particle using Gaussian distribution (8):

$$
P\left(X_{i, t} \mid \mu_{t}, \sigma_{t}\right)=\frac{1}{\sqrt{2 \sigma_{t}^{2} \pi}} e^{-\left(x-\mu_{t}\right)^{2}} / 2 \sigma_{t}^{2}
$$

Here, $\mu_{t}$ and $\sigma_{t}$ is determined by quality of WiFi / GPS location estimation and $X_{i, t}$ is estimated location of particle $\mathrm{i}$ at current time t. While Dilution of precision (DOP) of GPS can be used to estimate these two variables when GPS is available, the corresponding values for WiFi are fixed and estimated through evaluation of empirical experiment results. This fusion architecture is interesting since it allows the system to switch between GPS signal and WiFi estimation effortlessly. Since WiFi Fingerprinting has constant estimated error, switching between GPS and WiFi is decided by comparing quality of available GPS to WiFi estimation. Hence, a stable result is expected from the fusion system.

$$
\begin{gathered}
P\left(X_{i, t} \mid \mathrm{s}_{t}\right)=P\left(X_{i, t} \mid \mu_{t}, \sigma_{t}\right) S\left(X_{i, t}\right) \\
E_{t}=\sum_{n}^{i=0} P\left(X_{i, t} \mid \mathrm{s}_{t}\right) X_{i, t}
\end{gathered}
$$

With particle evolution model using odometer sensors data and covariance updating from $\mathrm{WiFi} / \mathrm{GPS}$, the estimated particles will represent results from traditional SLAM motion model component. A multinomial resampling [11], [12] of particles is required at this stage to refine particles pool. Significant particles will be brought to matching and scoring step with laser 
data in the current position. Equation (9) shows how SLAM score $S\left(X_{i, t}\right)$ for matching process of each particle will be fused with score from WiFi/ GPS covariance correction. By using WiFi/ GPS expected errors as covariance of fusion, particles will tend to be distributed around $\mathrm{WiFi} / \mathrm{GPS}$ reference. Finally, an estimation of current position is made by normalize score all particles and take mean value as shown in (10).

\section{EXPERIMENTS AND RESULTS}

Experiments are carried out in INRIA Rocquencourt campus using a cyber car and a version of Credibilist SLAM [1]. The cyber car (Fig. 3) is equipped with one front IbeoLux LIDAR sensor, a standard $2.4 \mathrm{GHz} \mathrm{WiFi}$ antennas for WiFi Fingerprinting and an IMU for odometer information. A RealTime Kinematic GPS (RTK GPS) antenna is mounted on the car to give precise ground truth.

The cybercar is then guided through test paths as shown in Fig. 4. There are four intersections noted on the map: A, B, C, D. Test paths are sequenced as follows: $A-B-C-D-B-A$. Path from $\mathrm{A}-\mathrm{B}$ with sufficient WiFi infrastructure will be trained for WiFi Fingerprinting localization. A standard GPS with expected error of 6 meters is utilized in combination with SLAM. There are 2 experimental scenarios:

(1): A fusion of standard GPS and Credibilist SLAM path: A-B-C-D-B-A.

(2): A combination of WiFi/standard GPS and Credibilist SLAM with WiFi and SLAM for A-B, B-A; Standard GPS and SLAM for B-C-D-B. Here, the path from A-B is simulated for poor-GPS case where WiFi Fingerprinting is available. The system is then tested for the ability to replace GPS with WiFi information and vice versa.

Before integrating $\mathrm{WiFi}$ into the system, it is necessary to investigate the characteristic and expected error bound of $\mathrm{WiFi}$ alone as a localization method. This is done by another experiment along the entire test path A-B. For this path, a radio map is learned including 15 reference points. At each point, 30 scans of WiFi signal are collected as training data. An ensemble of 50 neural networks, each with 170 input neurons, 90 nodes at hidden layer and 15 outputs are trained. Average of predictions from all networks will then be calculated. A threshold of 0.55 is set for which prior probability from prediction must overcome to be counted as a valid localization estimate. Fig. 4 shows an independent $\mathrm{WiFi}$ localization result with particle filter. It proves that alone, WiFi localization is able to track vehicle. The Euclidian error of each localization output is calculated and presented in Fig. 5. The average error in entire path is 3.328 meters and $98 \%$ of errors are under 6 meters. This allows us to set $\sigma_{W i F i}$ at 6 meters for fusion equation.

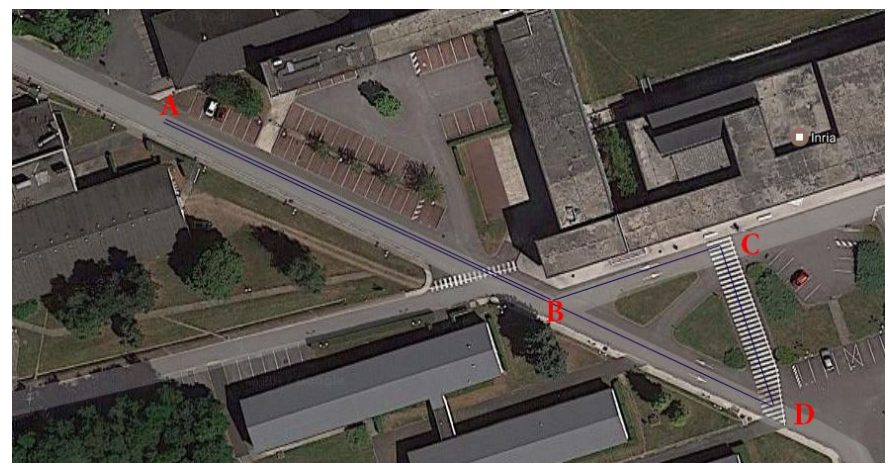

Fig. 2. Experiment environment - INRIA Rocquencourt campus

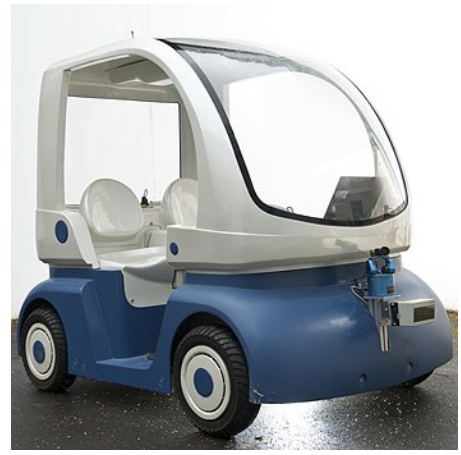

Fig. 3. Cybercar-RITS team INRIA

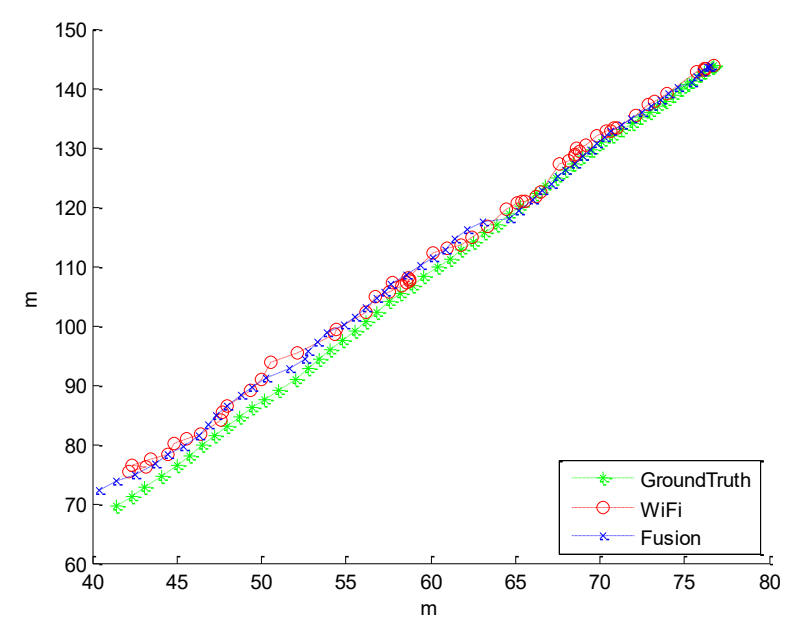

Fig. 4. WiFi localization result (red) and fusion with SLAM (blue) 


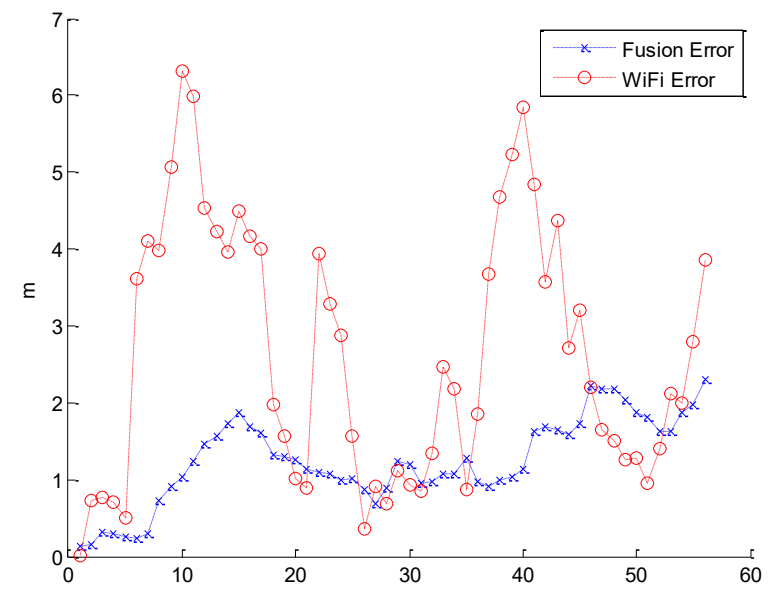

Fig. 5. Error distribution of WiFi localization and fusion with SLAM

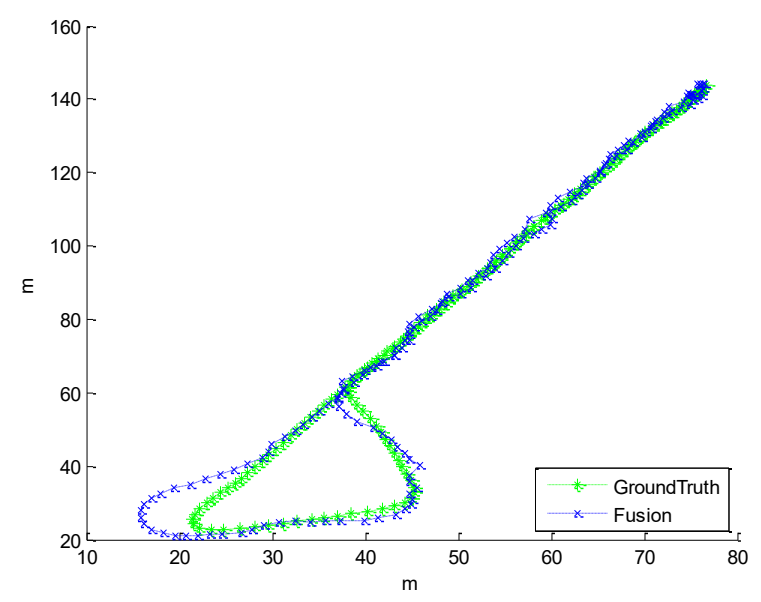

Fig. 6. Fusion of standard GPS and SLAM $\left(\sigma_{G P S}=6\right)$

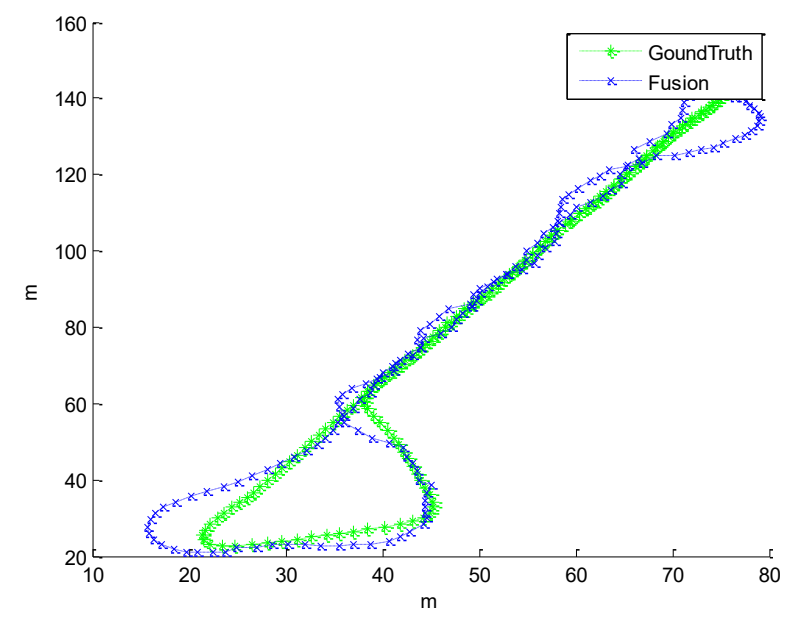

Fig. 7. Fusion of weak GPS and SLAM $\left(\sigma_{G P S}=10\right)$

In the first scenario, GPS information is assumed to be available for whole path A-B-C-D-B-A. Fig. 6 shows a RTK GPS ground truth in green and a fusion of SLAM and standard
GPS in blue. With assists from GPS, SLAM can recognize the previous location and follow the vehicle with only one LIDAR sensor setup.

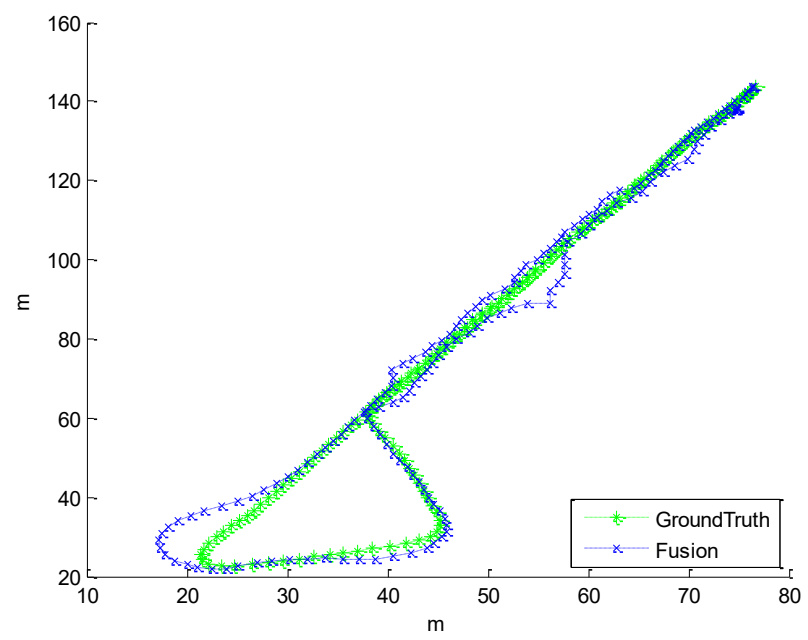

Fig. 8. Fusion of WiFi, Standard GPS and SLAM $\left(\sigma_{G P S}=6, \sigma_{W i F i}=6\right)$

In the second scenario, the path from A-B is assumed to be a weak GPS area. The vehicle is required to activate $\mathrm{WiFi}$ localization for A-B then replace it with GPS when possible (BC-D). Localization result is shown in Fig. 7 with the green ground truth of RTK GPS and the blue fusion localization result of WiFi, GPS and SLAM. In this case, the average error of fusion localization method is estimated at 2.7 meters with maximum error of 8 meters.

\section{CONCLUSION}

This paper presents an alternative solution for weak GPS area with WiFi Fingerprinting localization technique. A fusion strategy for WiFi, GPS and SLAM are proposed to adapt the system to the change of environmental conditions seamlessly. Early results show that a combination of WiFi and SLAM can be a replacement for weak GPS and SLAM fusion. In the future, several techniques will be applied to improve the system such as: a deep learning strategy for wifi fingerprinting as well as a multi-receiver setup for wifi fingerprints.

\section{ACKNOWLEDGMENTS}

Authors express their gratitude to the French project VALET and the RITS Team for its support in the development of this work.

\section{REFERENCES}

[1] G. Trehard, Z. Alsayed, E. Pollard, B. Bradai, and F. Nashashibi, "Credibilist simultaneous Localization and Mapping with a LIDAR," IEEE Int. Conf. Intell. Robot. Syst., pp. 2699-2706, 2014.

[2] J. Levinson, M. Montemerlo, and S. Thrun, "Map-Based Precision Vehicle Localization in Urban Environments," Robot. Sci. Syst. III, pp. 121-128, 2008.

[3] S. Rezaei and R. Sengupta, "Kalman Filter-Based Integration of DGPS and Vehicle Sensors for Localization," vol. 15, no. 6, pp. 1080-1088, 2007 
[4] S. Wahl, P. Schlumberger, R. Rojas, and M. Stampfle, "Localization inside a populated parking garage by using particle filters with a map of the static environment," IEEE Intell. Veh. Symp. Proc., vol. 2015-Augus, no. Iv, pp. 95-100, 2015.

[5] C. Fouque, P. Bonnifait, D. Bétaille, and A. Working, "Enhancement of Global Vehicle Localization using Navigable Road Maps and DeadReckoning," pp. 1286-1291, 2008.

[6] R. Parker and S. Valaee, "Vehicle Localization in Vehicular Networks," pp. 0-4, 2006.

[7] N. M. Drawil and O. Basir, "Intervehicle-Communication-Assisted Localization," vol. 11, no. 3, pp. 678-691, 2010.

[8] A. Amini, R. M. Vaghefi, J. M. De Garza, and R. M. Buehrer, "Improving GPS-Based Vehicle Positioning for Intelligent Transportation Systems," no. Iv, pp. 1023-1029, 2014.
[9] L. Breiman, "Bagging Predictors," Mach. Learn., vol. 24, pp. 123-140, 1996.

[10] T. G. Dietterich, "Ensemble Methods in Machine Learning," Mult. Classif. Syst., vol. 1857, pp. 1-15, 2000.

[11] "ON RESAMPLING ALGORITHMS FOR PARTICLE FILTERS Jeroen D . Hol, Thomas B . Sch " on , Fredrik Gustafsson Division of Automatic Control Department of Electrical Engineering Link " oping University."

[12] D. J. Salmond, "Novel approach to nonlinear/non-Gaussian Bayesian state estimation," IEEE Proceedings F, Radar and Signal Processing, vol. 140, pp. 107-113, 1993. 